\title{
Reflectance of Metal Grid Lines Calculated by Free Path Theory
}

\author{
YanRu Chen ${ }^{1}$, ShiMeng Feng ${ }^{2 *}$ \\ ${ }^{1}$ School of Mechanical Engineering, Shanghai JiaoTong University \\ ${ }^{2}$ Department of Physics and Astronomy, Shanghai JiaoTong University \\ *Email: smfeng@sjtu.edu.cn
}

\begin{abstract}
In this paper we present a model to investigate the reflectance on a metallic grid line. Based on the free path theory in the basic physics, we derived a corrected expression for calculating the free path in a metallic grid line. Then, we obtain a formula for describing the dependence of optical reflectance on its size. The theoretical results show that the size of grid line has a relatively large effect on its reflectance when the width and thickness of grid line are below $300 \mathrm{~nm}$. The reflectance will reach the value of bulk material when the width and thickness of grid line are over $800 \mathrm{~nm}$.
\end{abstract}

Keywords: Metallic grid line, mean free path, reflectivity

PACS: 78.67.Uh; 78.20.Ci; 78.20.-e; 74.62.Yb

\section{Introduction}

The reflectance of metallic wire grid is a very interesting and vexing problem. But in the past years, the research of grid line optical property mainly concentrated on the experimental study of its reflectance and polarized property[1-6]. For example, H. Blancher studied an experimental method to determine the wave-change of phase and its reflection on a grid line[7]. M Suzuki[8-9] successfully deposited one grid line polarizers by tuning the thicknesses of both the absorptive and the gap lavers with low reflectivity for visible light. An aluminum grid line polarizer had been fabricated on a quartz substrate by electron-beam direct-writing lithography, and a loss of less than 20\% and an extinction ratio larger than 1000 had been obtained [10]. So far, few papers were connected with the theoretical investigation of optical property on metallic grid line. A widely recognized method to calculate the reflectance has not been reported.

In this paper, based on the theory of reflectance on material surface, we present a model to investigate the reflectance of metallic grid line. We make great efforts to give a relatively accurate theoretical expression for describing the relationship of reflectance of grid line with its size, which is meaningful in applications in many fields.

\section{Theory}

The grid line is shown in Fig 1. We assume that the thickness of grid line is equal to the width for the convenient study.

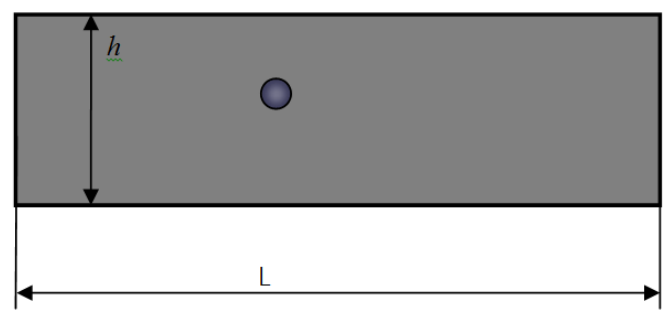

Figure 1. The model of grid line

As known well, the free electronic is considered as the free electronic gas in the metallic material. According to the collision theory of gas molecules, we have 


$$
\bar{z}=\sqrt{2} \pi d^{2} \bar{v} n
$$

Here $\bar{z}$ is the collision number per unit time of a free electron in the metallic bulk material, $d$ is the diameter of electronic, $n$ is the electronic density, $\bar{v}$ is the average speed of electronic. Thus, the total number of collision per unit time among all electronic is written as

$$
N=V n \bar{z}=V \sqrt{2} \pi d^{2} \bar{v} n^{2}
$$

Here $V$ is the volume of metallic grid line, $N$ is the total number of collision per unit time between arbitrarily two electronic. At the same time, the collision number between the electron and the surface of metallic grid line is

$$
N_{1}=S \frac{1}{4} n \vec{v}
$$

Here $S$ is the surface area of metallic grid line, $N_{1}$ is the collision number between the electron and the surface of grid line. Because the surface collision can affect the free path of electronic, so the total number of collision per unit time $\left(N_{0}\right)$ in the grid line should be written as

$$
N_{0}=S \frac{1}{4} n \vec{v}+V \sqrt{2} \pi d^{2} \bar{v} n^{2}
$$

Using $\mathrm{Eq}(4)$, the average collision number per unit time of a free electron should be corrected as the following

$$
\bar{Z}^{\prime}=\frac{N_{0}}{V n}=\frac{S \frac{1}{4} n \bar{v}+V \sqrt{2} \pi d^{2} \bar{v} n^{2}}{V n}=\frac{S \frac{1}{4} \vec{v}+V \sqrt{2} \pi d^{2} \bar{v} n}{V}
$$

Here $\bar{Z}^{\prime}$ is the corrected average collision number. Based on the definition of electronic free path, the corrected electron free path $(\bar{\lambda})$ in the grid line can be written as

$$
\bar{\lambda}=\frac{\bar{v}}{\bar{z}}=\frac{V}{S \frac{1}{4}+V \sqrt{2} \pi d^{2} n}
$$

Using $\lambda_{0}=\frac{1}{\sqrt{2} \pi d^{2} n}$ (defined as the free path of free electronic in the bulk material), $\mathrm{Eq}(6)$ can be changed into

$$
\bar{\lambda}=\frac{\lambda_{0}}{\left(\frac{S}{4\left(V \sqrt{2} \pi d^{2} n\right)}+1\right)}
$$

Here $\bar{\lambda}$ is the corrected free path in the grid line. Because the conductivity is proportional to the free path, the corrected conductivity can also be expressed as

$$
\sigma=k \bar{\lambda}=\bar{\lambda}=\frac{k \lambda_{0}}{\left(\frac{S}{4\left(V \sqrt{2} \pi d^{2} n\right)}+1\right)}
$$

Here $k$ is a proportional constant, using $\sigma_{0}=k \lambda_{0}$, Equation (8) can also be written as:

$$
\sigma=\frac{\sigma_{0}}{\frac{S}{4 V \sqrt{2} \pi d^{2} n}+1}
$$

Here $\sigma_{0}$ is the electrical conductivity in the bulk metal, $\sigma$ is the corrected electronic conductivity in the grid line. For the grid line with thickness $=h$, width $=h$, length $=\mathrm{L}$, respectively, we get

$$
\left\{\begin{array}{l}
V=L h^{2} \\
S=4 L h
\end{array}\right.
$$


Here $V$ is the volume of the metallic grid line, $S$ is the surface area of the corresponding grid line. Subsisting (10) into (9), we have

$$
\sigma=\frac{\sigma_{0}}{\frac{(4 L h)}{4 L h^{2} \sqrt{2} \pi d^{2} n}+1}=\frac{\sigma_{0}}{\frac{1}{h \sqrt{2} \pi d^{2} n}+1}
$$

Using $\lambda_{0}=\frac{1}{\sqrt{2} \pi d^{2} n}$ again, Eq (11) can be changed into

$$
\sigma=\frac{\sigma_{0}}{\frac{1}{h \sqrt{2} \pi d^{2} n}+1}=\frac{\sigma_{0}}{\frac{\lambda_{0}}{h}+1}
$$

Using Eq(12), we give the relationship cure of conductivity with the size of grid line shown in Fig.2. For gold, silver and copper, the free path in the bulk material are $\lambda=40 \mathrm{~nm}, \lambda=30 \mathrm{~nm}, \lambda=20 \mathrm{~nm}, \sigma_{0}=0.02065$ $\left(\Omega \cdot \mathrm{mm}^{2} / \mathrm{m}\right)$ of gold and $\sigma_{0}=0.0159\left(\Omega \cdot \mathrm{mm}^{2} / \mathrm{m}\right)$ of silver, and $\sigma_{0}=0.0167\left(\Omega \cdot \mathrm{mm}^{2} / \mathrm{m}\right)$ of copper.

Fig.2 (a) corresponds to cure of (12) when $h$ varies from 100 and $1000 \mathrm{~nm}$. From Fig.2 (a), the size of grid line has an effect on its conductivity. When the thickness and width of grid line vary from 100 and $300 \mathrm{~nm}$, it's conductivity will increase with the increase of thickness and width. But when the thickness and width of the grid line change from 400 and $800 \mathrm{~nm}$, its conductivity will slowly increase with the increase of thickness and width. Fig.2(b) shows the relation curve of Eq (12) when $h$ varies from 100 and $10000 \mathrm{~nm}$. Seeing from Fig. 2 (b), when the thickness and width of grid line exceed $800 \mathrm{~nm}$, its conductivity will be very close to that of bulk material.

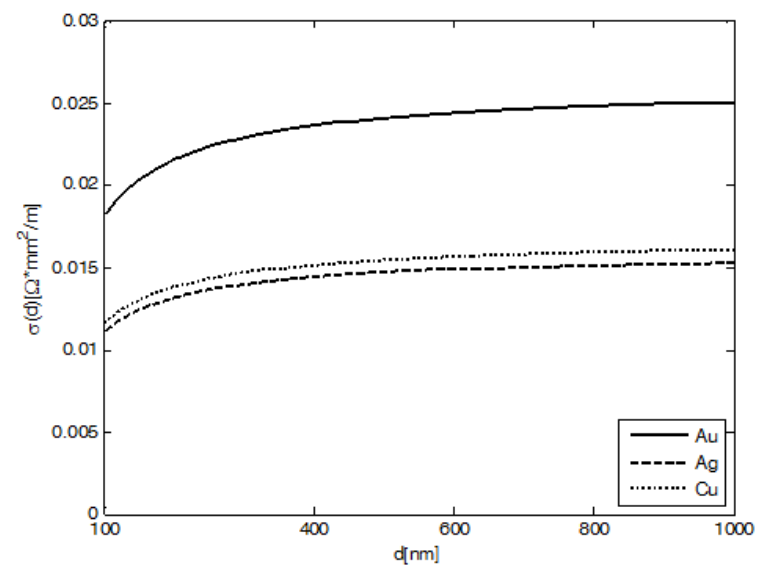

(a)

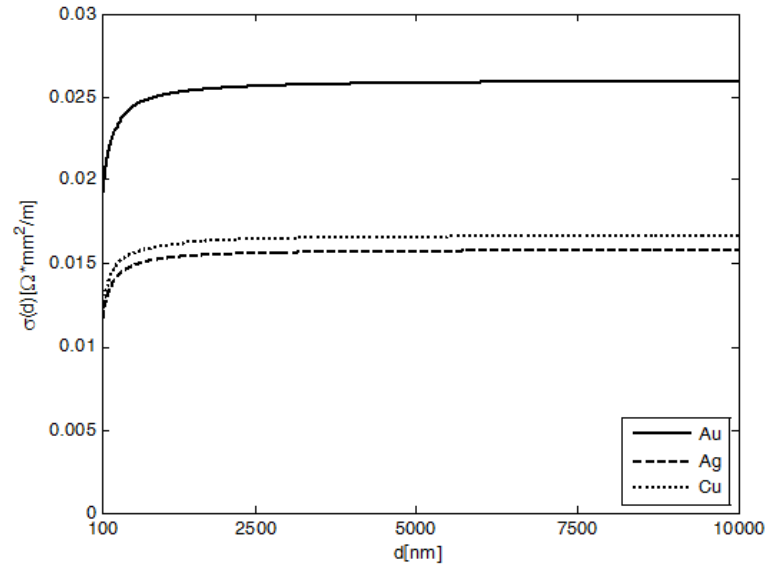

(b)

Figure 2. The conductivity curve of grid line in different size of line

\section{The Reflectance of Grid Line}

According to the basic theory, the relationship of refractive index with the conductivity is

$$
\tilde{\varepsilon}=\varepsilon_{\mathrm{r}}+i \frac{\sigma}{\varepsilon_{0} \omega}
$$

Here $\tilde{\varepsilon}$ is the complex dielectric constant of metal material, $\varepsilon_{\mathrm{r}}$ is the relative dielectric constant, $\varepsilon_{0}$ is the dielectric constant in vacuum, $\omega$ is the angular frequency of light. Using $n=n_{0}+i k=\sqrt{\varepsilon_{\mathrm{r}}+i \frac{\sigma}{\varepsilon_{0} \omega}}$, we can obtain, respectively, 


$$
n_{0}=\sqrt{\frac{1}{2}\left\{\left(\varepsilon_{r}^{2}+\left(\frac{\sigma}{\omega \varepsilon_{0}}\right)^{2}\right)^{\frac{1}{2}}+\varepsilon_{r}\right\}}, k=\sqrt{\frac{1}{2}\left\{\left(\varepsilon_{r}^{2}+\left(\frac{\sigma}{\omega \varepsilon_{0}}\right)^{2}\right)^{\frac{1}{2}}-\varepsilon_{r}\right\}}
$$

Here $n_{0}$ is the real part of refractive index, $k$ is the imaginary part. According to the reflectivity formulation, we have

$$
R=\frac{\left(n_{0}-1\right)^{2}+k^{2}}{\left(n_{0}+1\right)^{2}+k^{2}}=\frac{\left(\sqrt{\frac{1}{2}\left\{\left(\varepsilon_{r}^{2}+\left(\frac{\sigma}{\omega \varepsilon_{0}}\right)^{2}\right)^{\frac{1}{2}}+\varepsilon_{r}\right\}}-1\right)^{2}+\frac{1}{2}\left\{\left(\varepsilon_{r}^{2}+\left(\frac{\sigma}{\omega \varepsilon_{0}}\right)^{2}\right)^{\frac{1}{2}}-\varepsilon_{r}\right\}}{\left(\sqrt{\frac{1}{2}\left\{\left(\varepsilon_{r}^{2}+\left(\frac{\sigma}{\omega \varepsilon_{0}}\right)^{2}\right)^{\frac{1}{2}}+\varepsilon_{r}\right\}}+1\right)^{2}+\frac{1}{2}\left\{\left(\varepsilon_{r}^{2}+\left(\frac{\sigma}{\omega \varepsilon_{0}}\right)^{2}\right)^{\frac{1}{2}}-\varepsilon_{r}\right\}}
$$

Substitution of (12) into (15) produces

$$
R=\frac{\left(\sqrt{\frac{1}{2}\left\{\left(\varepsilon_{r}^{2}+\left(\frac{\sigma_{0}}{\omega \varepsilon_{0}\left(\frac{\lambda_{0}}{h}+1\right)}\right)^{2}\right)^{\frac{1}{2}}+\varepsilon_{r}\right\}}-1\right)^{2}+\frac{1}{2}\left\{\left(\varepsilon_{r}^{2}+\left(\frac{\sigma_{0}}{\omega \varepsilon_{0}\left(\frac{\lambda_{0}}{h}+1\right)}\right)^{2}\right)^{\frac{1}{2}}-\varepsilon_{r}\right\}}{\left(\sqrt{\frac{1}{2}\left\{\left(\varepsilon_{r}^{2}+\left(\frac{\sigma_{0}}{\omega \varepsilon_{0}\left(\frac{\lambda_{0}}{h}+1\right)}\right)^{2}\right)^{\frac{1}{2}}+\varepsilon_{r}\right\}}+1\right)^{2}+\frac{1}{2}\left\{\left(\varepsilon_{r}^{2}+\left(\frac{\sigma_{0}}{\omega \varepsilon_{0}\left(\frac{\lambda_{0}}{h}+1\right)}\right)^{2}\right)^{\frac{1}{2}}-\varepsilon_{r}\right\}}
$$

For silver, $\lambda=40 \mathrm{~nm}, \sigma_{0}=0.0159\left(\Omega \cdot \mathrm{mm}^{2} / \mathrm{m}\right)$ in the bulk material. When the light wavelength is equal to $632 \mathrm{~nm}$ and $532 \mathrm{~nm}$, respectively, the corresponding relative dielectric constant of metal (Ag) $\varepsilon_{r}=15,9.8$, respectively. Fig. 3 is the reflectance cure of metallic grid line at the wavelength of $532 \mathrm{~nm}$ and $632 \mathrm{~nm}$, respectively.

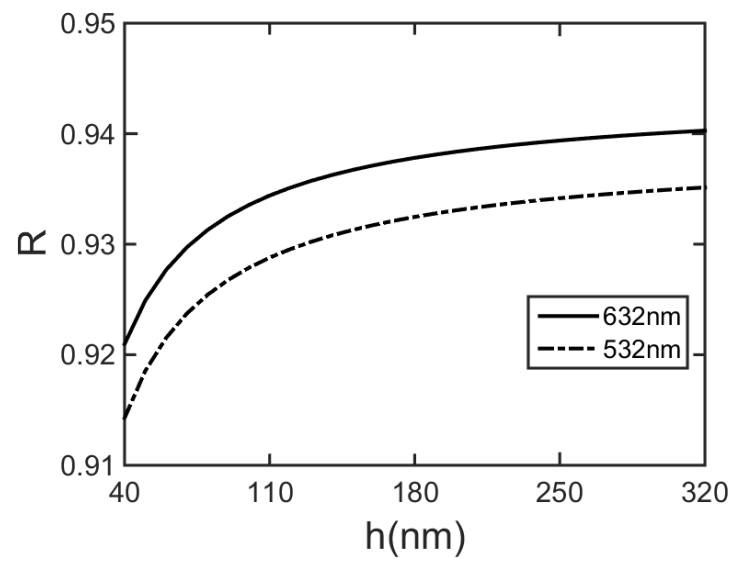

(a)

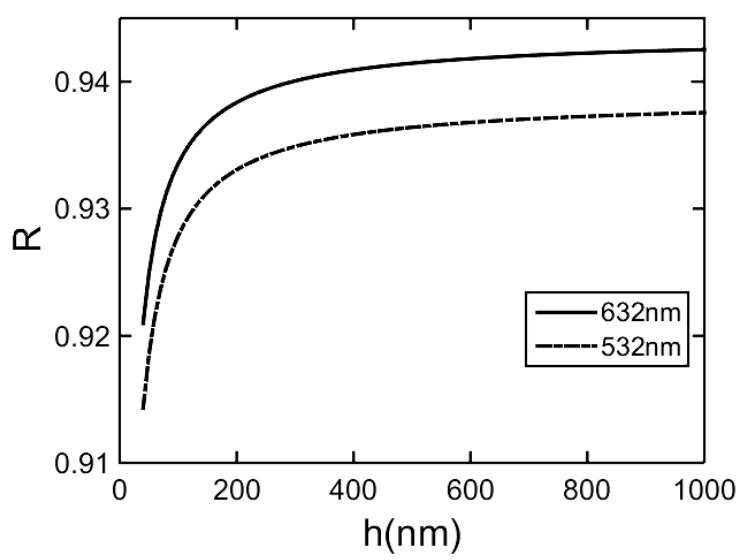

(b)

Figure 3. The relationship cure of the reflectance with the width of Ag grid line 
Fig.3 (a) corresponds to cure of (16) when $h$ varies between 40 and $400 \mathrm{~nm}$. Seeing from Fig.3 (a), when $h$ varies between 40 and $300 \mathrm{~nm}$, its reflectance will rapidly increase with the increase of $h$. But when $h$ of the grid line changes between 400 and $800 \mathrm{~nm}$, its reflectance will slowly increase with the increase of $h$. Fig.3(b) shows the curve of Eq (16) when $h$ varies from 40 and $1000 \mathrm{~nm}$. When $h$ of grid line exceeds $800 \mathrm{~nm}$, the reflectance will reach to the reflectance of bulk material.

In a word, the width and thickness have an effect on the optical property of grid line when they are small.

\section{Conclusions}

Based on the reflectance theory on the metallic material, we present a model to calculate the reflectance of metal wire grid. At first, we derive a corrected formula to describe the relationship of free path with the grid line size. Further, a corrected expression describing the relationship of the reflectance with the size of metal grid line is given. The theoretical results demonstrate that the size of metallic grid line can affect its reflectance under small width and thickness.

\section{References}

1. G.Cincotti, Polarization gratings design and applications, IEEE Journal of Quantum Electronics, 2004, 39(12):1645-1652

2. M.Suzuki, A.Takada, T.Yamada, T.Hayasaka, K.Sasaki, Low-reflective wire-grid polarizers with absorptive interference overlayers, Nanotechnology, 2010, 21 (17):175604

3. B.Schnabel, E.Kley, F.Wyrowski, Study on polarizing visible light by subwavelength -period metal-stripe gratings, Optical Engineering, 1999, 38(2):220-226

4. A. R. Rennie. Comment on the inverse polarization effect in metal-stripe polarizers, Journal of Optics a Pure \& Applied Optics, 2001, 3(1):67-71(5)

5. A. I. Utkin, A. A. Yushkanov, The effect of reflectance coefficients on the interaction of an $H$ wave with a thin metal film, Optics and spectroscopy, 117 (4)( 2014) 632-635

6. A.Takada,K. Sasaki, E.Takahashi, N.Hanashima, T.Yamada, Low reflective Wire grid Polarizers with an Absorptive Layer Formed by Glancing Angle Deposition, Journal of the Institute of Image Information, 2011, 65:1440-1445

7. H. Blancher R. Occelli J. M. Moynault H. Blancher R. Occelli J. M. Moynault, Optical properties of F.I.R. metallic grids: Reflection phase shift measurements International. Journal of Infrared and Millimeter Waves, November 1991, Volume 12, Issue 11, 1309-1314

8. M.Suzuki, Low-reflectivity wire-grid polarizers multilayered by the glancing-angle -deposition technique, Journal of Nanophotonics, 2011, 5 (1):1501

9. M.Suzuki, A.Takada, T.Yamada, T.Hayasaka, K.Sasaki, Antireflection coatings with FeSi layer: Application to low-reflectivity wire grid polarizers, Thin Solid Films, 2011, 519(24):8485-8489

10. H.Tamada, T.Doumuki, T.Yamaguchi, S.Matsumoto, Al wire-grid polarizer using the polarization resonance effect at the 0.8um-wavelength band, Optics Letters,1997, 22 (6):419 